\title{
A Rare Case of Rib Osteomyelitis in an Intravenous Drug Abuser
}

\author{
Jeffrey Song*, Tariq Sharman \\ Department of Internal Medicine, Southern Ohio Medical Center, Portsmouth, U.S.A. \\ *Corresponding author: j_song11@hotmail.com
}

Received May 13, 2019; Revised June 22, 2019; Accepted July 08, 2019

\begin{abstract}
Osteomyelitis (OM) is one of the most common invasive bacterial infections. Rib osteomyelitis is a rare disease that occurs in $\sim 1 \%$ or less of all osteomyelitis. It can result from direct inoculation from traumatic wounds, by spreading from adjacent tissue affected by cellulitis or septic arthritis, or through hematogenous seeding. We present a case of a 32-year-old female with a history of Intravenous drug abuse who presented with localized left-sided chest pain. A CT thorax contrast study was obtained that showed edema around the anterior aspects of the left 3rd to fifth ribs and involves the inferior border of the pectoralis muscle with buckling of the left 4th rib with fluid density in the fascial planes. Subsequently, an MRI was done that revealed the pathological fracture of the anterior aspect of the left forth rib with evidence of osteomyelitis of the left 4th and 5th ribs, as well as early insufficiency fracture of the left fifth rib. Blood cultures were negative. She was treated conservatively with empiric antibiotics due to lack of seeding and bacteremia.
\end{abstract}

Keywords: rib osteomyelitis, osteomyelitis

Cite This Article: Jeffrey Song, and Tariq Sharman, "A Rare Case of Rib Osteomyelitis in an Intravenous Drug Abuser.” American Journal of Medical Case Reports, vol. 7, no. 8 (2019): 158-159. doi: 10.12691/ajmcr-7-8-1.

\section{Introduction}

Osteomyelitis (OM) is one of the most common invasive bacterial infections. However, rib osteomyelitis is a rare disease that occurs in less than $1 \%$ of all cases of osteomyelitis [2]. It can result from direct inoculation from traumatic wounds, spreading from adjacent tissue affected by cellulitis, septic arthritis or through hematogenous seeding [1]. We are presenting a rare case of Rib Osteomyelitis in a patient with active intravenous drug abuse.

\section{Case Presentation}

The patient is a 32-year-old Caucasian female with history of active intravenous drug abuse, prior Methicillin-resistant Staphylococcus aureus bacteremia complicated with tricuspid valve endocarditis, who presented to our facility with complaints of left-sided chest pain, localized to the left $3^{\text {rd }}$ and 4rd ribs, sharp in nature with radiation to her back for 3 days. On examination, she is afebrile, normotensive, with normal pulse. She is non-toxic appearing, but uncomfortable from pain. She does not have any palpable lymphadenopathy. Her heart rhythm is regular with no murmurs, and breath sounds are bilaterally coarse. Examination of the affected region reveals no significant erythema, and no skin breakages. However, there is an area of visible depression in the soft tissue in a horizontally linear fashion around the $3^{\text {rd }}$ and $4^{\text {th }}$ ribs. It is exquisitely tender with no fluctuance. Her laboratory studies reveal mild microcytic anemia with no leukocytosis. Metabolic panel is within normal limits. Procalcitonin is elevated at $0.51 \mathrm{ug} / \mathrm{L}$. Alkaline Phosphatase is normal. Erythrocyte sedimentation rate is elevated at 74 millimeter/hour. C-Reactive Protein is also elevated at $7.28 \mathrm{mg} / \mathrm{dL}$. A Computed tomography (CT) of the thorax with contrast was obtained that showed edema around the anterior aspects of the left $3^{\text {rd }}$ to fifth ribs and involves the inferior border of the pectoralis muscle with buckling of the left $4^{\text {th }}$ rib with fluid density in the fascial planes. Subsequently, a Magnetic Resonance Imaging (MRI) was done that revealed the pathological fracture of the anterior aspect of the left $4^{\text {th }}$ rib with evidence of osteomyelitis of the left $4^{\text {th }}$ and $5^{\text {th }}$ ribs, as well as early insufficiency fracture of the left $5^{\text {th }}$ rib (see Figure 1 and Figure 2). She was empirically started on Intravenous Vancomycin and Intravenous Ceftriaxone. Her blood cultures showed no growth after 72 hours. A surgical consultation was obtained, who suggested conservative treatment with antibiotics as the area is difficult to access with significant morbidity. The patient was subsequently discharged with close follow-up imaging with oral minocycline and ciprofloxacin. 


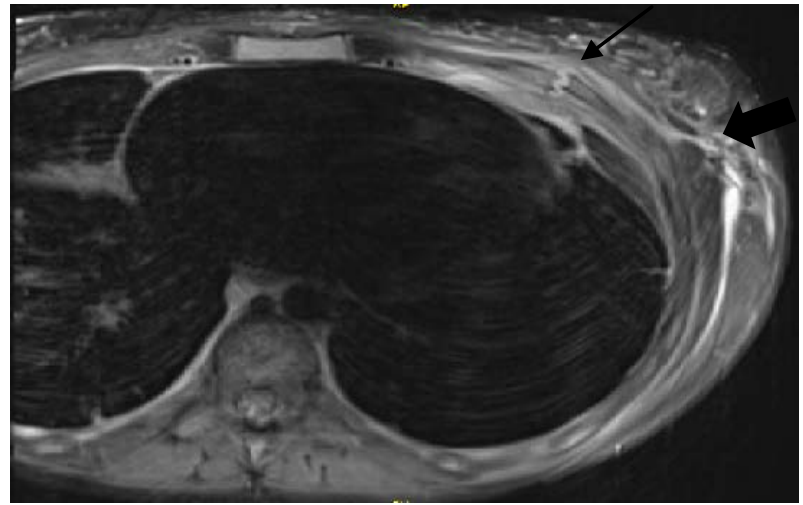

Figure 1. MRI showing buckle fracture seen at the anterior aspect of the left fourth rib (line arrow) with anterior displacement of the lateral fracture (block arrow)

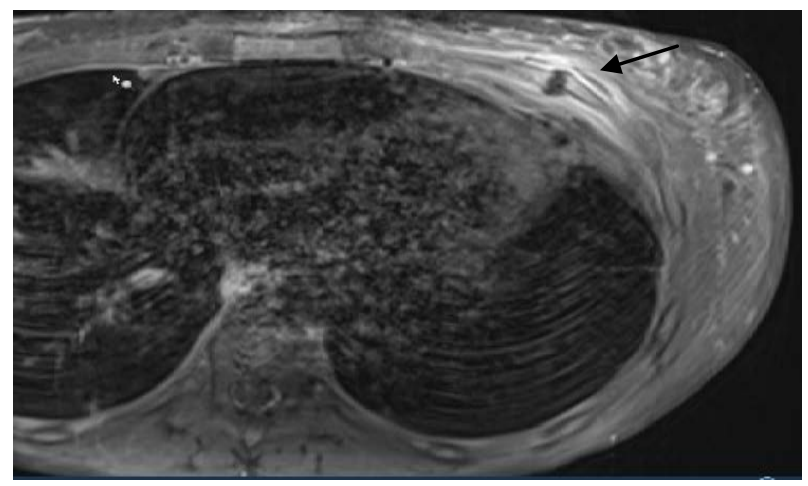

Figure 2. MRI showing enhancement of the affected overlying soft tissues with a thin layer of fluid along the anterior border of the fractured rib (arrow)

\section{Discussion}

Rib osteomyelitis is a rare condition in industrialized countries, with most cases occurring in children and young adults with predisposing conditions such as lung empyema, trauma of the chest, chronic granulomatous diseases, and sickle cell anemia [1,2]. Route of infection is mostly from contiguous spread, followed by hematogenous route of infection [1]. The causative organism of rib osteomyelitis is primarily mycobacterial infections followed by bacterial infections. Intravenous drug abuse is certainly a high-risk factor of infections, including infections of the skin and soft-tissue, septic arthritis, osteomyelitis, and endovascular infections [2]. The mean duration of symptoms before diagnosis is 16 days for osteomyelitis of bacterial etiology, with common clinical symptoms to include fever (73\%), soft tissue mass (64\%), and chest pain (60\%) [3]. Due to vague early symptoms, the diagnosis can often be delayed potentially leading to complications such as pathological fractures, swelling, abscesses and bacteremia [4]. Treatment of rib osteomyelitis is similar to other types of osteomyelitis and includes prolonged antibiotic therapy with possible surgical intervention. In our patient with no evidence of bacteremia nor seeding, surgical intervention was deferred as it was felt that the risks and morbidities associated with accessing this difficult area outweighed the potential benefits. Empiric antibiotics were initiated with considerations of bioavailability, adequate coverage and feasibility. Due to our patient's habit of intravenous drug abuse, we elected not to use parenteral antibiotics. Though gram-negative osteomyelitis and soft tissue infections are not common, it may certainly need to be considered in drug addicts. Oral Ciprofloxacin has been shown to be biochemically achieved in bone samples, especially with higher doses and infected bone samples [5]. Our patient was discharged on this regimen with close outpatient follow-up and repeat imaging.

\section{Conclusion}

In conclusion, rib osteomyelitis is a rare entity that can be easily misdiagnosed because of its rarity and non-specific clinical signs. Clinicians should have a high index of suspicion especially in a patient who abuses intravenous substances.

\section{References}

[1] [Osteomyelitis of the ribs in the antibiotic era. Bishara, J, et al. 2000, Scand J Infect Dis, pp. 32(3): 223-7.

[2] Bacterial Infections in Drug Users. Gordon, Rachel and Lowy, Franklin . 2005, N Engl J Med, pp. 353:1945-1954.

[3] Salmonella Osteomyelitis of Ribs. Khan, Imran, et al. 2018, Journal of Ayub Medical College Abbottabad-Pakistn, p. 30(4).

[4] Escherichia coli rib osteomyelitis. Matsuura, H, et al. 2019, QJM: An International Journal of Medicine, pp. Volume 112, Issue 1, January 2019; 35-36.

[5] Ciprofloxacin Concentrations in Bone and Muscle after Oral Dosing. Fong, Ignatius W, et al. March 1986 (405-408), s.l. : Antimicrobial Agents and Chemotherapy, 1985.

[6] Osteomyelitis of sternum and rib after breast prosthesis implantation: A rare or underestimated infection? Seng, Piseth, et al. 2015, IDCases, pp. 2(1): 31-33.

(C) The Author(s) 2019. This article is an open access article distributed under the terms and conditions of the Creative Commons Attribution (CC BY) license (http://creativecommons.org/licenses/by/4.0/). 